\title{
Natural rubber foams with anisotropic cellular structures: Mechanical properties and modeling
}

\author{
Leandra Oliveira-Salmazo ${ }^{\mathrm{a}, \mathrm{b}, *}$, Alberto Lopez-Gil $^{\mathrm{a}}$, Felipe Silva-Bellucci ${ }^{\mathrm{c}}$, Aldo E. Job $^{\mathrm{b}}$, \\ Miguel A. Rodriguez-Perez ${ }^{\text {a }}$ \\ a Cellular Materials Laboratory, CellMat, Condensed Matter Physics Department, University of Valladolid, Paseo de Belén 7, 47011 Valladolid, Spain \\ ${ }^{\mathrm{b}}$ Faculdade de Ciências e Tecnologia, UNESP-Universidade Estadual Paulista, Rua Roberto Simonsen, 305, Presidente Prudente, São Paulo CEP 19060-900, \\ Brazil \\ c Ministério da Ciência, Tecnologia e Inovação-MCTI, Rua: Esplanada dos Ministérios, Bloco E, 01, CEP 70067-900 Brasília-DF, Brazil
}

\section{A R T I C L E I N F O}

\section{Article history:}

Received 9 June 2015

Received in revised form 27 October 2015

Accepted 28 October 2015

Available online 6 December 2015

\section{Keywords:}

Biopolymer

Natural rubber

Cross-linked

Anisotropy

Foams

\begin{abstract}
A B S T R A C T
Natural rubber foams are biobased and lightweight products which have found their main field of application in comfort products such as mattresses and pillows. They are generally produced from chemical foaming processes in which the expansion of the polymer is isotropic and hence, their properties are not directionally dependent. However, this dependency could be interesting for certain structural and thermal insulating applications. In this work, elastomeric foams based on natural rubber with a medium relative density (around 0.3 ) and with varied cellular structures in terms of the shape anisotropy ratio of the cells were produced by a chemical foaming process in which expansion was restricted to only one direction inside a mold. The use of solid precursors of different dimensions, the elastomeric properties of natural rubber and the crosslinking by sulfur of the polymer matrix during foaming allowed foams to be obtained with anisotropy ratios between 0.90 and 2.48 at the same density and with the same properties as the polymeric matrix. In this particular case the study was focused on analysing their compressive modulus and its relationship with the anisotropy of the cellular structure by employing analytical models generally used to describe the mechanical behavior of anisotropic foams.
\end{abstract}

(c) 2015 Elsevier B.V. All rights reserved.

\section{Introduction}

Natural rubber is a biobased elastomeric polymer which has been mainly applied in the tire industry. As foam, natural rubber is generally used for comfort applications such as in mattresses and pillows. These foams are produced from latex, which is the liquid form of natural rubber and directly extracted from the Hevea brasiliensis tree (Ferreira, 2002; Kanokwiroon et al., 2008). However, the homogeneity in properties of latex is not always guaranteed due to the high amount of substances found in it, such as proteins, fatty acids, carbohydrates, phospholipids and inorganic salts (Archer, 1963). Moreover, latex requires chemical stabilization by ammonia prior to being processed because its properties can be affected by the environment during storage. On the contrary, dry natural rubber, which is obtained from latex after centrifugation and drying, is more stable and homogeneous in properties (Najib

\footnotetext{
* Corresponding author at: University of Valladolid, Paseo de Belén 7, Valladolid 47011, Spain. Fax: +34 983423192.

E-mail address: lesalmazo@yahoo.com.br (M.A. Rodriguez-Perez).
}

et al., 2011). Natural rubber foams produced from dry natural rubber have great potential for being employed in several industrial sectors such as in the aeronautics and automotive industries, sports equipment, footwear, toys and packaging.

Natural rubber has been studied in detail as the main polymer matrix in biobased composites (Jong, 2015; Wu et al., 2004) and nanocomposites (Abrahama et al., 2013; Bellucci et al., 2012). However, there are not many works in literature dealing with the production of dry natural rubber foams. The few ones found employed chemical blowing agents, different grades of natural rubber and in some cases the properties of the polymer matrix were modified by the addition of carbon black (Ariff et al., 2008; JoonHyung et al., 2006, 2007; Najib et al., 2009, 2011). All these works have in common that dry natural rubber is firstly chemically crosslinked by compression molding and later foamed at temperatures above the decomposition temperature of the blowing agent by free expansion. This produces cellular structures which are mainly isotropic. In this way, the properties of the foams obtained are not directionally dependent. The latex foams used in mattresses and pillows are obtained by industrial processes such as Talalay and 
Dunlop in which the expansion of the polymer is also isotropic (Eaves, 2004).

The production of natural rubber foams with anisotropic cellular structures could be important for applications in which for instance, different mechanical properties depending on the load direction are required. This is because the cells are elongated. Shape anisotropy in cells can be defined as the ratio between the maximum and the minimum length of the cell. Polymer foams usually present anisotropy ratios of about 1.3 (Gibson and Ashby, 1997). In literature there are several models that attempt to describe the elastic response of polymeric foams under a compressive load (Christensen, 1986; Gibson and Ashby, 1997; Zhu et al., 1997; Warren and Kraynik, 1987) such as the cubic cell model of Gibson and Ashby (1997). Nevertheless, in most of them anisotropic cells are not taken into account. Huber and Gibson (1988) later modified the model of the cubic cell with the aim of describing the behavior of anisotropic foams. They assumed a rectangular open cell instead of a cubic open cell and introduced the anisotropy ratio in the equation of the model. These models simulate the mechanical behavior of foams with very simple cell geometries such as cubes and rectangular prisms, which on many occasions do not represent the real morphology of the cells. A more complicated cell geometry is the tetrakaidecahedron, which is known as the Kelvin cell model (Thomson, 1887). It is assumed to be the only polyhedron that packs to fill space and minimize the surface area per unit volume. Several authors employed elongated tetrakaidecahedron with the aim of analyzing non-isotropic foams (Gong et al., 2005a,b). Sullivan et al. (2008) (Sullivan and Ghosn, 2009) defined a more general model than the previous authors based on the elongated tetrakaidecahedron by specifying three independent dimensions and introducing an additional shape parameter called $Q$. In these works of Sullivan et al., the stiffness and strength ratios of several flexible and rigid foams with anisotropy ratios between 1 and 1.8 are compared with the results obtained with the modified Kelvin model. Moreover, in the work of Hamilton et al. (2013) low-density reinforced polyurethane foams with anisotropy ratios between 1 and 2 were evaluated by the rectangular cell model and the modified Kelvin model.

For these reasons, the objectives of this work are firstly, the development of a new foaming route which allows elastomeric foams to be obtained from dry natural rubber and with different anisotropy ratios at a given density. Secondly, the study of the relationship between process, structure and properties of the foams produced. This study is mainly focused on their anisotropic morphology. The last objective is to evaluate if models generally used for low density foams such as the rectangular and Kelvin cell models are suitable for flexible foams of medium density and with anisotropy ratios higher than 2 such as the natural rubber foams developed in this work.

\section{Materials and production process}

\subsection{Materials}

Dry natural rubber of the type Crepe Brazilian Clear (CCB) was employed as the polymer matrix. The vulcanizing agents were zinc oxide supplied by Silox, stearic acid from Renichem, 2-mercaptobenzothiazole provided by Sigma-Aldrich and finally sulfur, which was supplied by Panreac. The blowing agent employed was azodicarbonamide Porofor MC-1 provided by Lanxess with an average particle size of $3.9 \mu \mathrm{m}$ and with a decomposition temperature of $210^{\circ} \mathrm{C}$ (data obtained from the technical data sheet of this material).

The formulation employed for the production of the vulcanized natural rubber foams is summarized in Table 1.
Table 1

Formulation.

\begin{tabular}{llc}
\hline Raw material & Chemical formula & $\mathrm{Phr}^{\mathrm{a}}$ \\
\hline Dry natural rubber & $\left(\mathrm{C}_{5} \mathrm{H}_{8}\right)_{\mathrm{n}}$ & 100 \\
Zinc oxide & $\mathrm{ZnO}$ & 4.25 \\
Stearic acid & $\mathrm{CH}_{3}\left(\mathrm{CH}_{2}\right)_{16} \mathrm{COOH}$ & 3 \\
Sulfur & $\mathrm{S}_{8}$ & 2 \\
2-Mercaptobenzothiazole & $\mathrm{S}_{2} \mathrm{NC}_{7} \mathrm{H}_{7}$ & 1 \\
Azodicarbonamide & $\mathrm{C}_{2} \mathrm{H}_{4} \mathrm{O}_{2} \mathrm{~N}_{4}$ & 5 \\
\hline
\end{tabular}

a Parts per hundred of natural rubber.

\subsection{Production process}

Three kinds of natural rubber foams with the same density but with different anisotropy ratios were produced in this work. The terminology employed for the different foams produced is NRFA (natural rubber foam of high Anisotropy), NRFM (natural rubber foam of Medium anisotropy) and NRFI (Isotropic natural rubber foam).

The production process of these foams consisted of 3 steps. In the first step, the raw materials were mixed in a Haake internal mixer model Rheodrive 5000. The temperature was set to $60^{\circ} \mathrm{C}$ and the screw speed to $60 \mathrm{rpm}$. The temperature was low enough to avoid premature crosslinking and decomposition of the blowing agent.

Secondly, the blends produced were thermoformed in a hotplates press to obtain solid precursors. The temperature employed was $85^{\circ} \mathrm{C}$ and the blends were pressed during $5 \mathrm{~min}$. Three kinds of cylindrical solid precursors of the same weight $(7 \mathrm{~g})$ but with different shape and dimensions were produced with the aim of obtaining the previously mentioned foams. The dimensions of the NRFA solid precursor were $10 \mathrm{~mm}$ in height and $31 \mathrm{~mm}$ in diameter. The NRFM solid precursor was $17.8 \mathrm{~mm}$ in height and $23.2 \mathrm{~mm}$ in diameter and finally, the NRFI solid precursor was $24.7 \mathrm{~mm}$ in height and $19.7 \mathrm{~mm}$ in diameter. These dimensions can be seen in Fig. 1 where 3D representations of both, the foaming mold and the solid precursors employed are shown.

Finally, the solid precursors obtained were placed in a cylindrical stainless-steel mold such as the one shown in Fig. 1 in order to carry out the final foaming step in which crosslinking and foaming took place simultaneously. A hot plates press supplied the heat required for both processes. The mold was placed between the plates of the press and the temperature was set to $160^{\circ} \mathrm{C}$. The plates of the press were just in contact with the upper and lower surfaces of the mold. After 25 min the mold was removed from the press and cooled down with water.

The height of the mold cavity was $32 \mathrm{~mm}$ as was its diameter. Therefore, the volume of the mold cavity was $25.7 \mathrm{~cm}^{3}$. The expected density for the natural rubber foams produced is $0.27 \mathrm{~g} / \mathrm{cm}^{3}$, which was calculated by dividing the mass of the solid precursors employed $(7 \mathrm{~g})$ by the volume of the mold cavity. The correspondent relative density is 0.29 . In Fig. 1 the terminology which has been used throughout the article is also shown with the aim of identifying the expansion direction $\left(D_{1}\right)$ and the directions perpendicular to it $\left(D_{2}\right.$ and $\left.D_{3}\right)$. Due to the cylindrical shape of the mold, directions $\mathrm{D}_{2}$ and $\mathrm{D}_{3}$ are equivalent and for that reason all the analyses were focused in one of these directions $\left(D_{2}\right)$.

\section{Experimental}

\subsection{Density and volumetric expansion ratio}

Density measurements of the foamed and solid samples were performed by the geometric method; that is by dividing the weight of each specimen between its corresponding volume (ASTM 


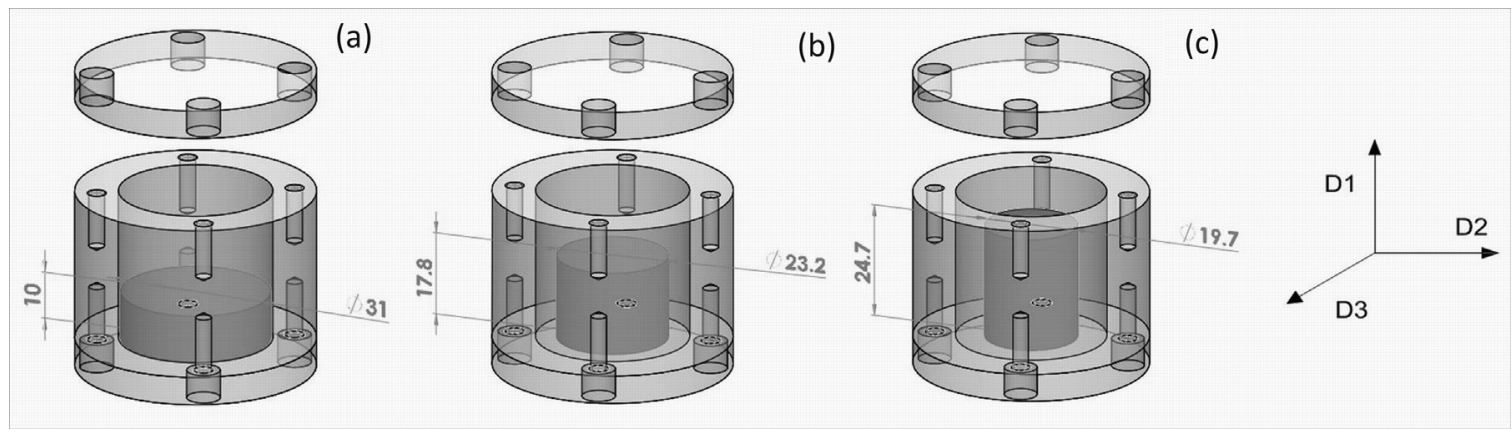

Fig. 1. Mold and solids precursors employed. (a) NRFA. (b) NRFM. (c) NRFI.

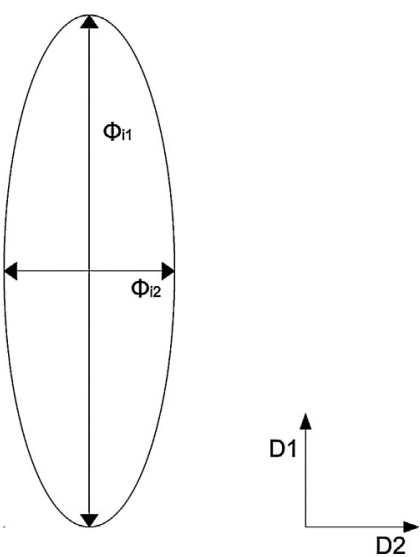

Fig. 2. Dimensions of the cells.

standard D1622-08). The volumetric expansion ratio (ER) was calculated as the inverse of the relative density as shown in Eq. (1).

$\mathrm{ER}=\frac{1}{\rho_{f} / \rho_{s}}$

where $\rho_{f}$ is the density of the foam, $\rho_{s}$ is the density of the solid polymer matrix $\left(0.93 \mathrm{~g} / \mathrm{cm}^{3}\right)$ and $\rho_{f} / \rho_{s}$ is the relative density.

\subsection{Cellular structure morphology}

The cellular structure of the samples was analyzed by using scanning electron microscopy images (SEM). First of all, samples were fractured after immersing them in liquid nitrogen. Secondly, they were made conductive by sputtering deposition of gold and finally observed using a Jeol JSM-820 scanning electron microscope. The SEM images were taken in the plane parallel to the expansion direction $\left(D_{1} / D_{2}\right)$ and in the plane perpendicular to it.

The cellular structure was characterized in the plane parallel to the expansion direction $\left(D_{1} / D_{2}\right)$ by using an image processing analysis tool based on the software Image J (Pinto et al., 2013). The size of each cell was expressed as the length of the cell in the expansion direction $\left(\Phi_{i 1}\right)$ and the length of the cell in a perpendicular direction to it $\left(\Phi_{i 2}\right)$ as shown in Fig. 2. The one-dimensional foam cell size in the expansion plane $\left(\Phi_{1,2}\right)$ was then calculated by Eq. (2) in which $n$ is the total number of cells. The two dimensional foam cell size in the expansion plane is expressed as the diameter of a theoretical sphere $(\bar{\Phi})$ and is calculated as the average between $\Phi_{1}$ and $\Phi_{2}$ (Eq. (3)) and the anisotropy ratio (R) was calculated as the ratio between these two values (Eq. (4)).

$\Phi_{1,2}=\frac{\sum_{i=1}^{n} \Phi_{i 1,2}}{n}$
$\bar{\Phi}=\frac{\Phi_{1}+\Phi_{2}}{2}$

$R=\frac{\Phi_{1}}{\Phi_{2}}$

A distribution of cell sizes in terms of $\Phi_{1}$ and $\Phi_{2}$ is represented with the aim of evaluating in more detail how the cell size varies depending on the solid precursor employed. The distribution is expressed as the frequency of cells in percentage within a range of cell sizes (bin size: $200 \mu \mathrm{m}$ ). The asymmetry coefficient (AC) for both, $\Phi_{1}$ and $\Phi_{2}$, was calculated by using the Eq. (5) where $\mathrm{SD}_{1,2}$ represents the standard deviation of the distribution in each direction, which is in turn calculated by Eq. (6).

$\mathrm{AC}_{1,2}=\frac{\sum_{i}^{n}\left(\Phi_{i(1,2)}-\Phi_{1,2}\right)^{3}}{n\left(\mathrm{SD}_{1,2}\right)^{3}}$

$\mathrm{SD}_{1,2}=\frac{\sqrt{\sum_{i}^{n}\left(\Phi_{i(1,2)}-\Phi_{1,2}\right)^{2}}}{n-1}$

The homogeneity of the cell size distributions is better described by the parameter NSD (normalized standard deviation) which is the ratio between SD and the cell size as expressed by Eq. (7).

$\mathrm{NSD}_{1,2}=\frac{\mathrm{SD}_{1,2}}{\Phi_{1,2}}$

Finally the cell density $\left(N_{o}\right.$, number of cells per cubic centimeter of the solid material) was calculated by Eq. (8). This calculus is based on the Kumarís theoretical approximation (Kumar, 1988; Weller and Kumar, 2010).

$N_{o}=\frac{N_{v}}{1-V_{f}}$

where $V_{f}$ is the volume fraction of voids (porosity) and $N_{v}$ is the number of cells per cubic centimeter of the foamed material. These parameters are calculated by Eqs. (9) and (10), respectively.

$V_{f}=1-\frac{\rho_{f}}{\rho_{s}}$

$N_{v}=\left[\frac{\left(n M^{2}\right)}{A}\right]^{3 / 2}$

In these equations $\rho_{f} / \rho_{s}$ is the relative density of the foamed material, $n$ is the number of cells analyzed, $A$ is the image area and $M$ is the magnification factor of the micrograph.

\subsection{Open cell content}

The percentage of open cells (OC) was measured with a gas pycnometer model AccuPyc II 1340 Micromeritics following the standard ASTM D2856-94. Cubic samples (about $10 \mathrm{~mm}$ on one 


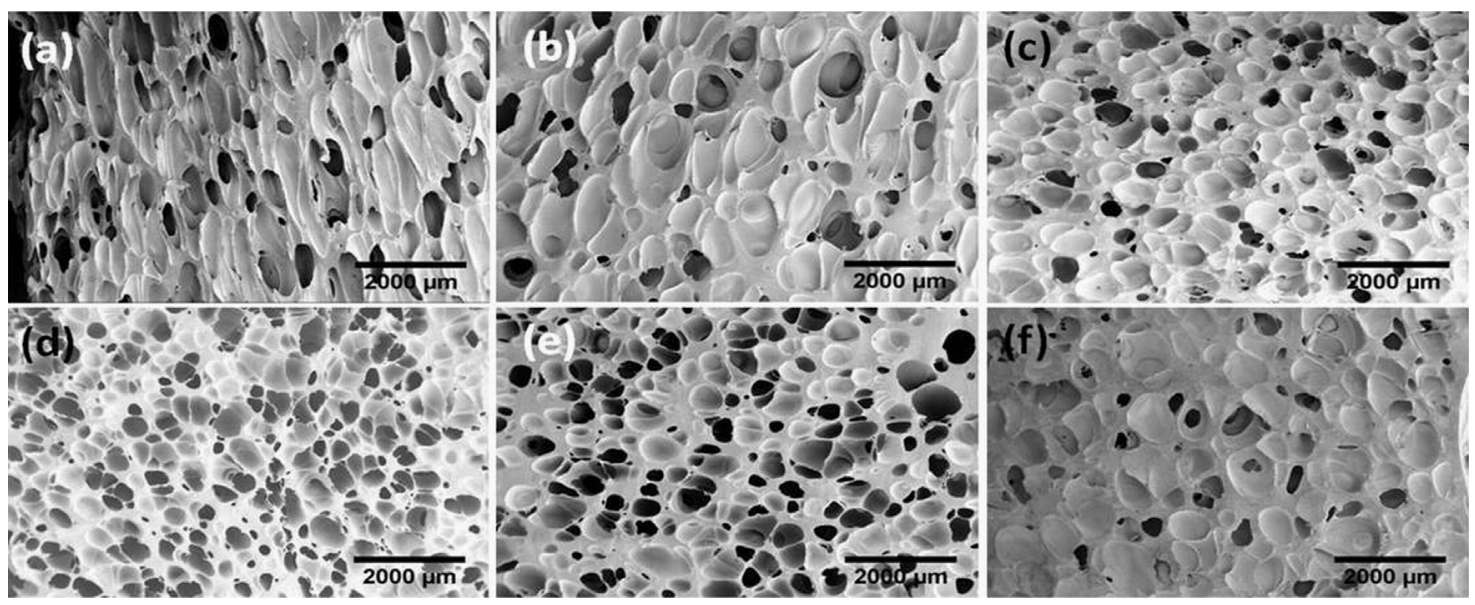

Fig. 3. SEM images of the foamed samples. Expansion plane: (a) NRFA. (b) NRFM. (c) NRFI. Perpendicular plane to the expansion direction. (d) NRFA. (e) NRFM. (f) NRFI.

side) extracted from each kind of foam were employed for the measurements and Eq. (11) was employed to calculate the open cell content:

$\mathrm{OC}=\frac{V-V_{1}-V_{s}}{V\left(V_{f}\right)}$

where $V$ represents the geometric volume of the sample, $V_{1}$ is the volume measured by the gas pycnometer and $V_{s}$ is the volume of the cells located at the surface which are open because the sample had to be cut in order to be extracted from the foam. $V_{s}$ was measured taking into consideration the cell size of the sample in the two main directions $\left(\Phi_{1}\right.$ and $\left.\Phi_{2}\right)$ as described in the standard ASTM D2856-94. Finally, $V_{f}$ is the porosity of the foamed sample which was previously described by Eq. (9).

\subsection{Compressive mechanical properties}

A mechanical characterization was carried out by compression tests. The stress-strain curves were obtained at room temperature with a Dynamic Mechanical analyzer model DMA-7, PerkinElmer with a capacity of up to $2.7 \mathrm{~N}$. The samples measured were cubes of $10 \mathrm{~mm}$ on one side and were the same ones employed for the open cell content measurements. Three replicates were measured with the aim of obtaining more accurate results. The foams were loaded not only in a plane perpendicular to the rise direction $\left(D_{1}\right)$ but also in a plane parallel to it. The stress-rate applied was $500 \mathrm{mN} / \mathrm{mm}$. The mechanical characterization was only performed in the elastic region and hence, the samples were loaded up to a stress level below the collapse strength of the foam. The compressive elastic modulus for both measurements $\left(E_{1}, E_{2}\right)$ was then calculated as the slope of the elastic-linear region of the stress-strain curves.

\section{Results and discussion}

\subsection{Cellular structure}

The cellular structure of the three kind of foams produced is shown in Fig. 3 by means of SEM images taken in a plane parallel to the expansion direction $\left(D_{1} / D_{2}\right)$ and in the plane perpendicular to it.

The SEM images taken in the expansion plane (Fig. 3a-c) show how the anisotropy of the cells varies depending on the geometry of the precursor employed. In the case of using precursors with practically the same diameter as the mold cavity (NRFA) the foam anisotropy ratio was higher than in the case of using solid precursors (NRFM and NRFI) whose diameter was lower than that of the
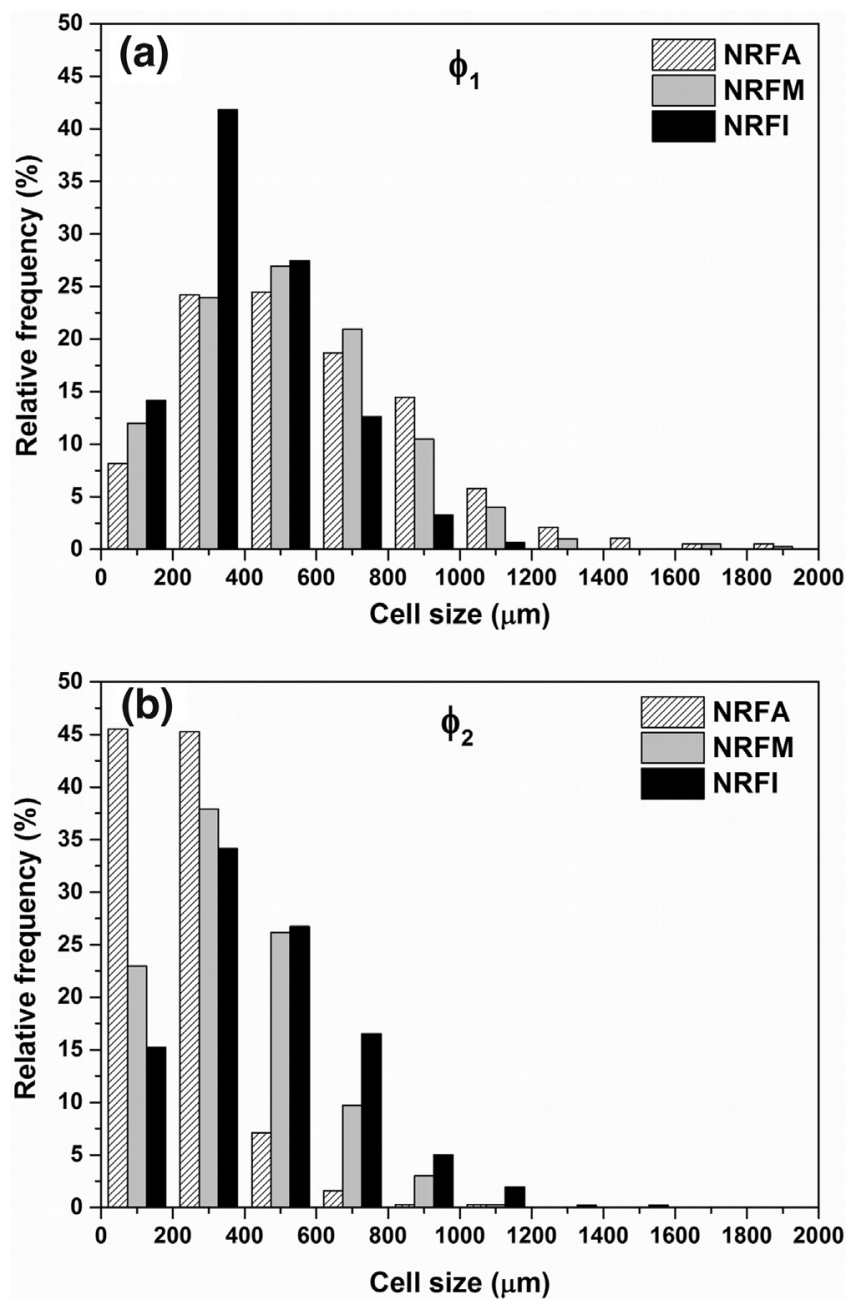

Fig. 4. Cell size distributions. (a) $\Phi_{1}$. (b) $\Phi_{2}$.

mold cavity. The SEM images correspondent to a plane perpendicular to the expansion direction (Fig. 3d-f) show cells practically isotropic which confirm that the cells present an axisymmetric geometry.

The cell size distribution for $D_{1}$ and $D_{2}$ is shown in Fig. 4 .

In the case of $\Phi_{1}$, the isotropic foam (NRFI) presents a more homogeneous distribution with most of the cells $(>65 \%)$ located in a range between 200 and $600 \mu \mathrm{m}$. On the contrary the anisotropic 
Table 2

Density and cellular structure parameters.

\begin{tabular}{llll}
\hline Parameters & NRFA & NRFM & NRFI \\
\hline$\rho_{f} / \rho_{s}$ & $0.339 \pm 0.018$ & $0.376 \pm 0.021$ & $0.364 \pm 0.080$ \\
$\mathrm{ER}$ & 2.95 & 2.66 & 2.74 \\
$\Phi_{1}(\mu \mathrm{m})$ & 584.1 & 532.1 & 401.4 \\
$\mathrm{NSD}_{1}$ & 0.56 & 0.53 & 0.49 \\
$\mathrm{AC}_{1}$ & 0.93 & 0.93 & 0.71 \\
$\Phi_{2}(\mu \mathrm{m})$ & 235.5 & 363.6 & 444.2 \\
$\mathrm{NSD}_{2}$ & 0.58 & 0.54 & 0.53 \\
$\mathrm{AC}_{2}$ & 1.45 & 0.66 & 0.77 \\
$-\Phi$ & 409.8 & 447.9 & 422.8 \\
$R$ & 2.48 & 1.46 & 0.90 \\
$N_{o}\left(\mathrm{cells} / \mathrm{cm}^{3}\right)$ & $9519.4 \pm 2492$ & $7664.5 \pm 2436$ & $10239.3 \pm 2145$ \\
$\mathrm{OC}(\%)$ & $44.1 \pm 4.2$ & $35.3 \pm 10.5$ & $52.4 \pm 1.0$ \\
\hline
\end{tabular}

foam (NRFA) presents a wider distribution than the isotropic foam. A high number of cells present values of $\Phi_{1}$ above $600 \mu \mathrm{m}$ (>40\%). In the case of $\Phi_{2}$ the anisotropic foam is concentrated mainly in the range of $0-200 \mu \mathrm{m}$ while the distribution for the less anisotropic foams is wider than the anisotropic one.

The relative density, the volumetric expansion ratio, the cell size in one dimension and in two dimensions $\left(\Phi_{1}, \Phi_{2}, \bar{\Phi}\right)$, their correspondent asymmetry coefficient (AC), normalized standard deviation (NSD), anisotropy ratio $(R)$, the cell density $\left(N_{0}\right)$ and the open cell content $(O C)$ are shown in Table 2.

The relative density as well as the volumetric expansion ratio is very similar for all the foams produced. This fact makes the comparison possible between the foams in terms of cellular structure because there is no influence of the density in the results obtained. The image analysis of the cellular structure in the expansion plane confirms the result that was observed in the SEM images. The anisotropy ratio varies depending on the solid precursor employed. Sample NRFA presents an average anisotropy ratio of 2.48 while the samples NRFM and NRFI present lower anisotropies, 1.46 and 0.90 , respectively. In spite of varying the anisotropy ratio the twodimensional cell size $(\bar{\Phi})$ did not vary significantly. Likewise with the $N_{o}$ values which are very similar for all the foams produced and therefore, this indicates that the cell nucleation rate and foaming kinetics are the same for each kind of foam. Regarding the onedimensional cell size, $\Phi_{1}$ and $\Phi_{2}$ are very similar in the case of the isotropic foam. However, these values start to be different as the anisotropy of the cells becomes higher. $\Phi_{1}$ grows and $\Phi_{2}$ decreases while increasing the anisotropy ratio. For instance, in the case of the anisotropic foam (NRFA) the value of $\Phi_{1}$ is $584.1 \mu \mathrm{m}$ while $\Phi_{2}$ is $235.5 \mu \mathrm{m}$. In general the distributions of cell sizes are homogeneous because the NSD values are below 1 and similar for all the materials. The shape of the distributions is the same for all the foams because the biggest cells are more separated from the average than the small ones as observed in Fig. 4. This is confirmed by the AC values which are all positive.

When considering the mechanical performance of foams of low or medium densities not only the cellular morphology but also the open cell content plays a key role. For this reason the open cell content was measured and later analyzed by means of SEM images. The values obtained are shown in Table 2 . The open cell content is in general very high and practically the same for all the foams produced. The high values obtained can be due to the fact that the foaming process takes place at the same time as the crosslinking of the polymer matrix and therefore, when reaching high expansions the molten polymer is not strong enough to support the pressure of the gas. This fact is confirmed by the SEM images shown in Fig. 5 where interconnections between cells can be seen.

The reason for obtaining different anisotropy ratios lies mainly in two facts: in the first place the foams were produced from solid precursors with the same geometry (all of them are cylinders) but of different dimensions. In the case of the NRFA foam the diameter of the solid precursor was practically the same as the mold while in the case of the other two foams (NRFM and NRFI) the diameter gradually decreased and at the same time the height increased. Secondly, the shape and dimensions of these solid precursors was not modified during the heating of the polymer up to the decomposition temperature of the blowing agent. This can be due on the one hand, to the elastomeric properties of dry natural rubber. It is an amorphous polymer whose glass transition temperature is below ambient temperature $\left(-60^{\circ} \mathrm{C}\right)$ and does not melt when the temperature is raised. On the other hand, the natural rubber is being cross-linked while the temperature increases and therefore the viscosity of the polymer matrix become higher. The combination of these two facts allows the solid precursor to start to expand without losing their original shape and dimensions.

Taking this fact into consideration the picture in Fig. 6 explains the evolution of the foaming process for each kind of foam from the point at which the blowing agent starts to decompose $\left(t_{0}\right)$. Several simplifications concerning a foaming process were assumed to make the schemes of Fig. 6: first of all the nucleation of cells is homogeneous; hence, the same number of cells was generated for each kind of foam at the same time. This was confirmed experimentally because the $N_{o}$ values are practically the same for all the foams produced as shown in Table 2. Secondly, during the expansion process $\left(t_{f}-t_{i}\right)$ there is no generation of new cells and all the gas produced goes into the cells already created at the beginning of the foaming process. Finally, the coalescence phenomenon was not taken into account. Under these restrictions and once the blowing agent starts to decompose $\left(t_{0}\right)$ the cells grow isotropically until the polymer reaches one of the mold walls $\left(t_{i}\right)$. In the case of the precursor whose diameter is practically the same as that of the mold (NRFA) this happens almost immediately. This implies that the expansion is restricted to only one direction earlier than in the other two foams $\left(t_{i \mathrm{NRFA}}<t_{i \mathrm{NRFM}}<t_{i \mathrm{NRFI}}\right)$ and the cells have more distance to grow anisotropically. But not only is there more distance but also more time for the anisotropic expansion to happen because $t_{f}$ is the same for all the foams and hence, $t_{f}-t_{i}$ for the NRFA foam it is higher than in the other two. For this reason the anisotropy ratio of the cells when the polymer completely fills the mold $\left(t_{f}\right)$ is higher in the NRFA foam than in the other two cases.

Taking the previous explanation into consideration a theoretical anisotropy ratio $\left(R_{t}\right)$ in two dimensions can be determined as the ratio between the foam height at $t_{f}\left(h_{f}\right)$ and the foam height at $t_{i}$ $\left(h_{i}\right)$. For this calculus it was supposed that during the anisotropic expansion (between $t_{i}$ and $t_{f}$ ) the length of the cells in the perpendicular direction $\left(D_{2}\right)$ was kept constant and they only grew in the expansion direction $\left(D_{1}\right)$. This is explained also in Fig. 6 where a $2 \mathrm{D}$ spherical cell is represented at $t_{i}$ (isotropic cell) and at $t_{f}$ (anisotropic cell). $x$ represents the length of the cell in $\mathrm{D}_{2}$ and $y$ represents the length of the cell in $\mathrm{D}_{1}$. This theoretical cell only grew in $\mathrm{D}_{1}$ during the anisotropic expansion and therefore, $x_{2}$ is the same as $x_{1}$ and at the same time is equal to $y_{1}\left(x_{1}=x_{2}=y_{1}\right)$. Due to this restriction, the problem is simplified to only one dimension because the only variation in dimensions takes place in $\mathrm{D}_{1}$ and therefore, the anisotropy ratio can be expressed theoretically as the ratio between $y_{2}$ and $y_{1}$ (Eq. (12)).

$R_{t}=\frac{y_{2}}{x_{2}}=\frac{y_{2}}{y_{1}}$

Assuming that the theoretical anisotropy ratio can be calculated as shown in Eq. (12), the ratio between the heights of the correspondent foams should lead to the same result. However, in this case the total number of cells in height has to be considered as shown in Fig. 6 where $h_{i}$ is the height of the foam at $t_{i}$ and $h_{f}$ is the height of the foam at $t_{f}$ (this was only represented in the NRFA sample but is applicable to the other samples as well). Therefore, 


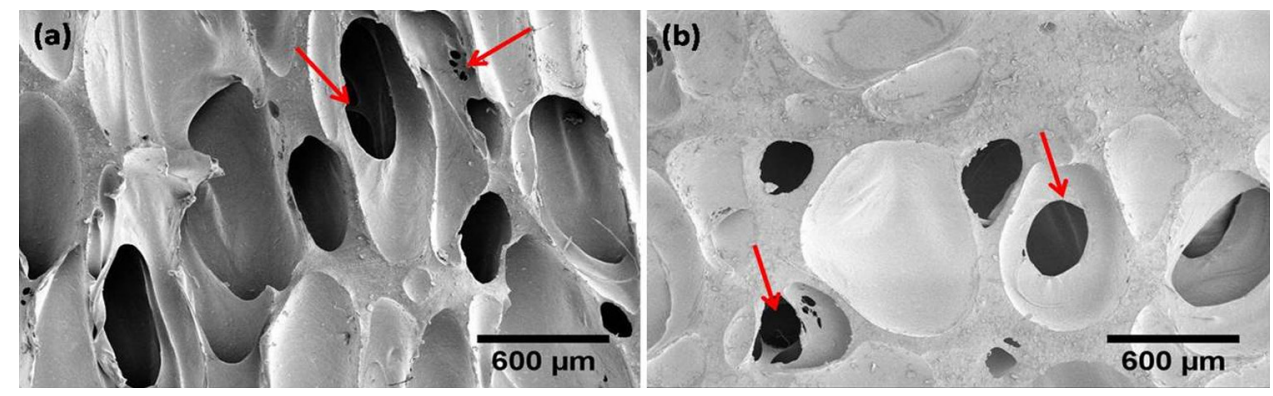

Fig. 5. SEM images of the foamed samples (40X). (a) NRFA. (b) NRFI.

(a)
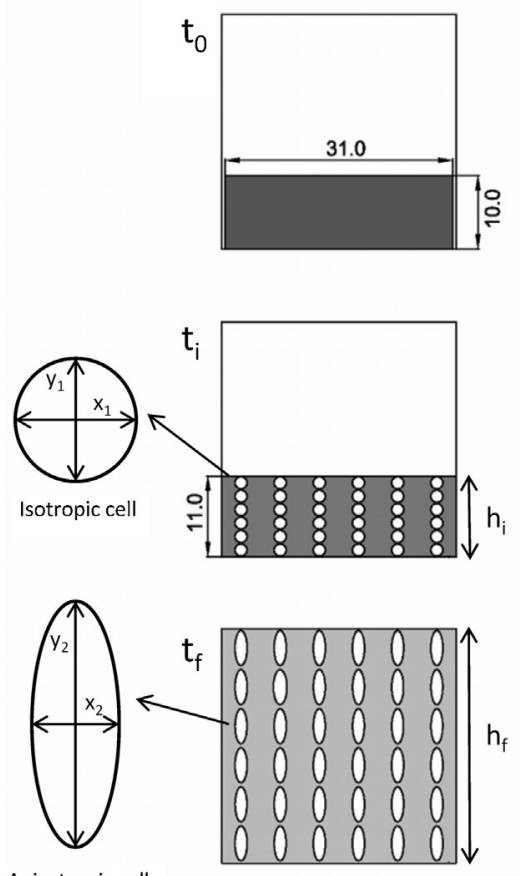

Anisotropic cell (b)
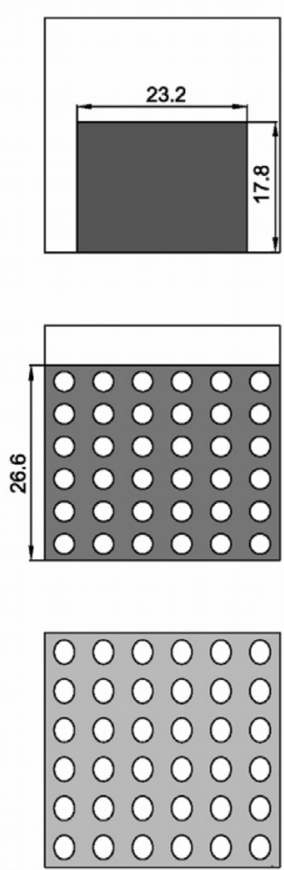

(c)

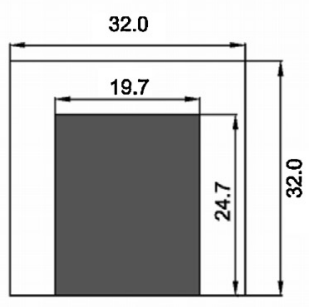

27.0
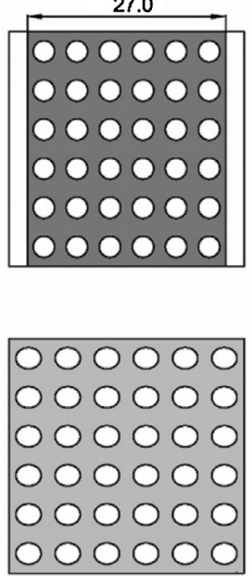

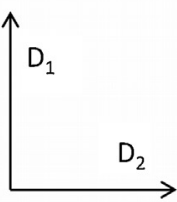

Fig. 6. Schematic representation of the expansion process of all the foams produced. (a) NRFA. (b) NRFM. (c) NRFI.

the number of cells in the expansion direction can be calculated as the ratio between $h$ and $y$ at the different times ( 6 cells in Fig. 6). Then, the ratio between $h_{f}$ and $h_{i}$ can be expressed by Eq. (13).

$\frac{h_{f}}{h_{i}}=\frac{y_{2} n_{f}}{y_{1} n_{i}}$

If it is assumed that there was no additional creation of cells $\left(n_{f}=n_{i}\right)$ during the anisotropic expansion of the polymer (between $t_{i}$ and $t_{f}$ ) and that the growth of the cells created at $t_{i}$ only took place in $\mathrm{D}_{1}\left(x_{2}=y_{1}\right)$, the Eq. (13) is simplified to Eq. (14) and the relationship between heights is equal to the theoretical anisotropy ratio.

$R_{t}=\frac{y_{2}}{x_{2}}=\frac{h_{f}}{h_{i}}$

These theoretical $\left(R_{t}\right)$ and experimental $\left(R_{e}\right)$ values are shown in Table 3 together with the precursor and foams dimensions.

The theoretical approach fits very well with the values obtained experimentally in the case of the NRFM and NRFI foams. However, in the case of the NRFA foam these values present a higher difference in the sense that $R_{t}$ is higher than $R_{e}$. This could be due to the fact that in this foam the time required for the anisotropic expansion $\left(t_{f}-t_{i}\right)$ is higher than in the other two kinds of foams and therefore, there are more options for new cells to be generated during this time. In this particular case $n_{f}$ would be higher than $n_{i}$ and $R_{t}$ should be calculated as indicated by Eq. (15). In this way the theoretical anisotropy ratio would be lower and probably more similar to the experimental result.

$R_{t}=\frac{y_{2}}{x_{2}}=\frac{h_{f} n_{i}}{h_{i} n_{p}}$

\subsection{Mechanical properties}

Mechanical compressive tests were performed on the foams produced in two directions: $\mathrm{D}_{1}$ and $\mathrm{D}_{2}$. Fig. 7 shows typical examples of the stress-strain curves obtained from the compression tests.

The compressive modulus of elasticity is calculated from these curves as the slope of the linear-elastic region. Fig. 8 represents the relative compressive modulus of all the foams produced in the two directions: $D_{1}$ and $D_{2}$. In spite of the fact that the density is very similar in all the cases, the compressive modulus is divided by the density with the aim of showing the results without its influence. 
Table 3

Theoretical and experimental anisotropy ratios.

\begin{tabular}{|c|c|c|c|c|c|}
\hline Sample & Solid precursor height $\left(h_{o}\right)$ & Foam height at $t_{i}\left(h_{i}\right)$ & Foam height at $t_{f}\left(h_{f}\right)$ & $R_{t}$ & $R_{e}$ \\
\hline NRFA & 10.0 & 11.0 & 32.0 & 2.91 & 2.48 \\
\hline NRFM & 17.8 & 26.6 & 32.0 & 1.20 & 1.46 \\
\hline NRFI & 24.7 & 27.0 & 32.0 & 0.84 & 0.90 \\
\hline
\end{tabular}

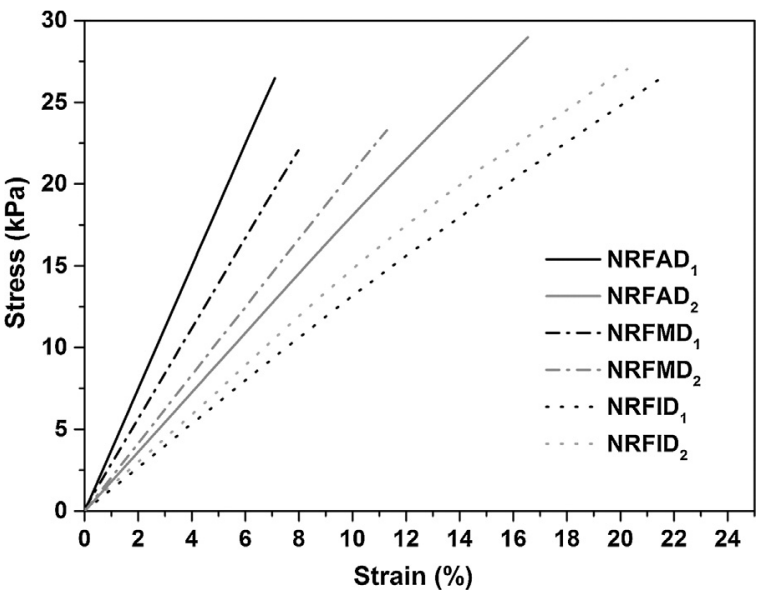

Fig. 7. Compressive stress-strain curves.

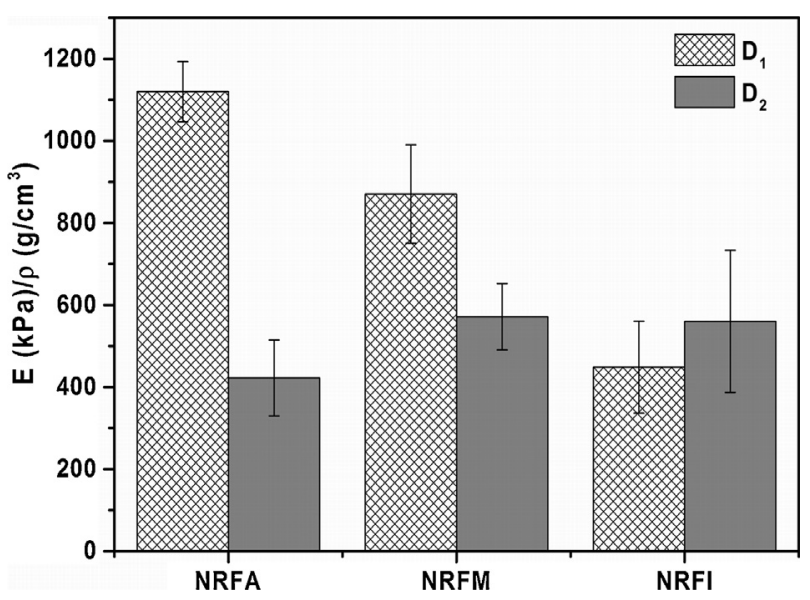

Fig. 8. Relative compressive modulus in two directions.

The most clear tendency is observed in the expansion direction $\left(D_{1}\right)$ in which the relative compressive modulus increases with the anisotropy ratio. The highest relative compressive modulus was obtained for the anisotropic foam (NRFA) whose experimental anisotropy ratio is 2.48 whereas the lowest was obtained for the isotropic foam (NRFI) whose experimental anisotropy ratio is 0.9 . The relative compressive modulus obtained for the direction $D_{2}$ is lower that the one obtained in $D_{1}$ confirming that these foams present an anisotropic cellular structure in which the cells are oriented in the expansion direction $\left(D_{1}\right)$. However, this does not happen in the NRFI foam in which the compressive modulus in $D_{1}$ is lower than that in $D_{2}$. This fact indicates that the cells can be oriented in another direction different from $\mathrm{D}_{1}$. This is confirmed by the cellular structure characterization because the experimental anisotropy ratio for this foam is below $1(0.9)$.

One of the objectives of this work is the comparison of experimental results for these medium density natural rubber foams with theoretical models found in literature that describe the mechanical behavior in the elastic region under compressive stresses of low density open cell polymeric foams. The first model cosiders a very

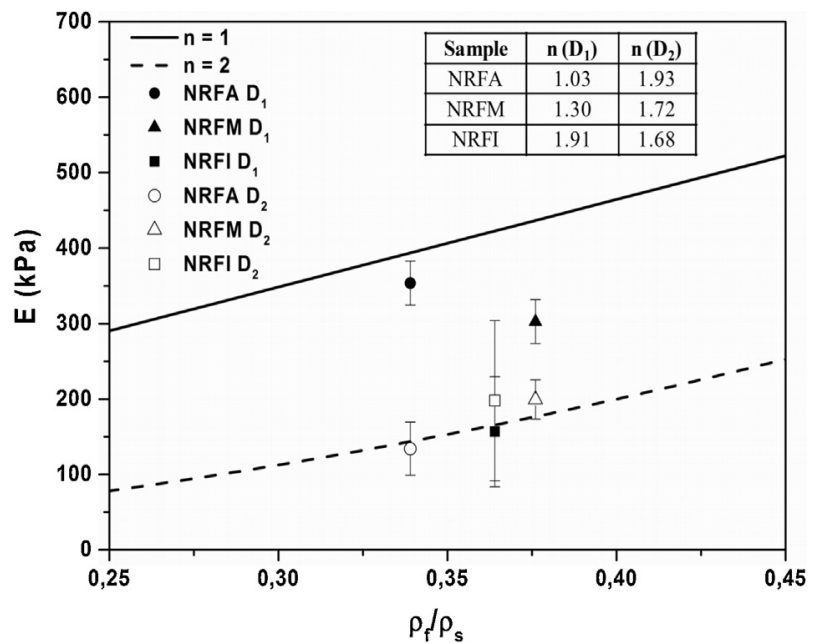

Fig. 9. Experimental values and theoretical predictions of the cubic cell model.

simple geometry because the cells are represented as open cubes. This is the model of Gibson and Ashby (1997) described by Eq. (16) in which the compressive modulus decreases as a function of relative density. It is fair to point out that in this model the anisotropy of the cellular structure is not considered.

$\frac{E_{f}}{E_{s}}=C\left(\frac{\rho_{f}}{\rho_{s}}\right)^{n}$

In this equation $E_{f}$ and $E_{s}$ are the compressive modulus of the foam and the solid respectively. The value of $E_{s}$ employed here was $1080 \mathrm{kPa}$ and it was obtained experimentally in a solid with the same crosslinking degree as the foam. $C$ is a constant of proportionality which is assumed to be 1 and $n$ is an exponent that mainly depends on the foam microstructure. The experimental values of compressive modulus and theoretical values for $n=1$ and for $n=2$ obtained by the cubic cell model are plotted in Fig. 9. The reason for choosing $n=1$ and $n=2$ is that most of the polymer based cellular materials have values of $n$ between these two limits (Huber and Gibson, 1988). Moreover, the experimental exponent was calculated for all the foams analysed and shown in the plot of Fig. 9.

Considering the expansion direction $\left(D_{1}\right)$, the lowest value of $n$ (1.03) was obtained for the anisotropic foam (NRFA) while the highest value (1.91) was obtained for the isotropic foam (NRFI).

In the second model, Huber and Gibson (1988) described a rectangular elongated open cell instead of an isotropic cubic cell. Eq. (17) is the one employed for this model and is the same as in the cubic cell model but introducing a function of the shape anisotropy ratio. In the case of loading the foam in the rise direction $\left(D_{1}\right)$ this function is $R$ which is defined as the ratio between $\Phi_{1}$ and $\Phi_{2}$. As shown in Eq. (17) there is a linear relationship between the anisotropy ratio and the compressive modulus of the foam.

$\frac{E_{f}}{E_{s}}=C\left(\frac{\rho_{f}}{\rho_{s}}\right)^{2} R$

The compressive modulus of the foam not only in the expansion direction $\left(E_{1}\right)$ but also in the direction perpendicular to it $\left(E_{2}\right)$, both of them divided by the square of relative density, are represented versus the anisotropy ratio in Fig. 10. The experimental results do 


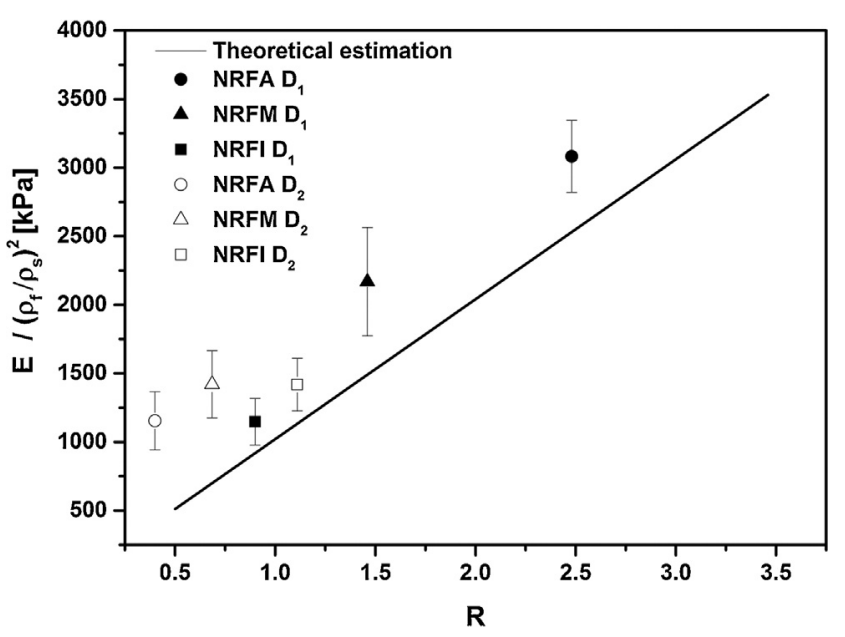

Fig. 10. Rectangular cell model. Theoretical and experimental values.

not fit accurately with the prediction of the model but the tendency is linear.

The rectangular cell model can be extended to measurements performed in two directions: $\mathrm{D}_{1}$ and $\mathrm{D}_{2}$. In this way, the calculus of theoretical values of the anisotropy ratio and the comparison with the experimental anisotropy ratio is possible. If the compressive modulus in the rise direction $\left(E_{1}\right)$ is divided by the compressive modulus in the other direction $\left(E_{2}\right)$ the relation obtained is only dependent on the anisotropy ratio as observed in Eq. (18).

$\frac{E_{1}}{E_{2}}=\frac{2 R^{2}}{1+\left(1 / R^{3}\right)}$

Fig. 11 represents $E_{1} / E_{2}$ versus the average anisotropy ratio. The black line represents the theoretical values obtained by the model. The white marks represent the experimental values for the natural rubber foams produced in this work. Moreover, other experimental values obtained from literature (Huber and Gibson, 1988) are represented with the aim of proving the validity of the model for polymeric foams.The tendency followed by the experimental results is different from that predicted by the model. The devia-

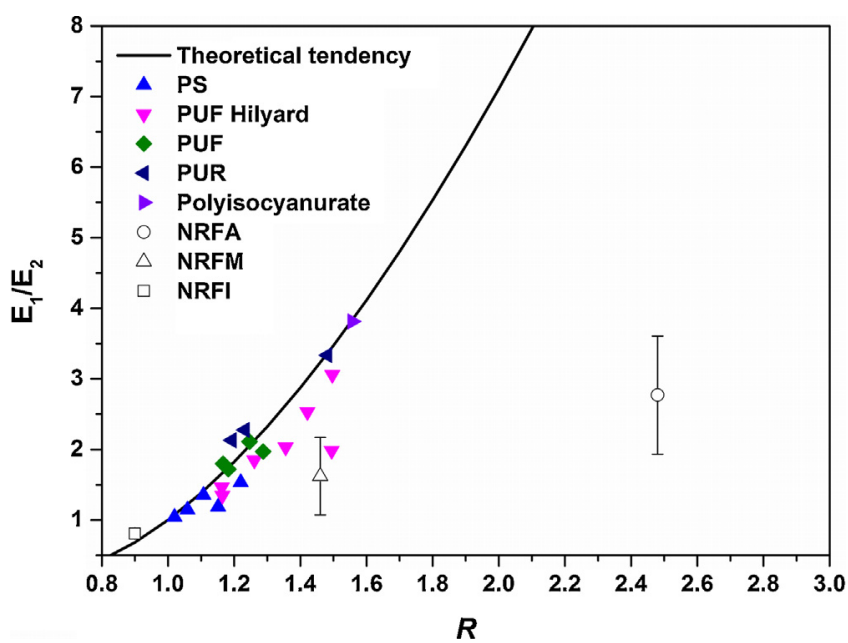

Fig. 11. Rectangular cell model. Theoretical and experimental values. ( $\mathbf{\Delta}) \operatorname{PS}($ Mehta and Colombo, 1976), ( ) PU(F) (Hilyard, 1982), ( ) PU(F) (Huber and Gibson, 1988)

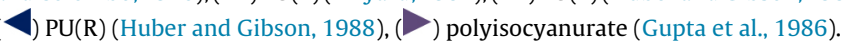

geometric term $Q$ that describes the morphology of the cell together with the shape anisotropy ratio $(R)$. Both parameters are independent. In addition, the relative density is also employed in the model. The Kelvin cell and the parameters that describe its morphology are reported in literature (Sullivan et al., 2008; Sullivan and Ghosn, 2009). Fig. 12 shows a 3D elongated tetrakaidecahedron which represents a typical Kelvin cell inside the correspondent theoretical rectangular cell. The main dimensions of this cell $(D, H, L, b$ and $\theta)$ are specified in the $2 \mathrm{D}$ representations.

$H$ is the length of the cell in the expansion direction and $D$ is the length of the cell in the direction perpendicular to it. $Q$ is calculated as the ratio between $b$ and $L \cos \theta$ as shown in Eq. (19).

$Q=\frac{b}{L \cos \theta}$

The ratio of the compressive modulus in the rise direction $\left(E_{1}\right)$ and the compressive modulus in the other direction $\left(E_{2}\right)$ is expressed by the Eq. (20).

$$
\frac{E_{1}}{E_{2}}=\frac{R^{2}}{4}\left[\frac{\left(2 \tilde{Q}^{2} R^{2}+64 Q^{3} / \sqrt{16+\tilde{Q}^{2} R^{2}}\right) C_{1}+8 R C_{2} \tilde{Q}^{3}\left(32+4 Q \sqrt{16+\tilde{Q}^{2} R^{2}}\right) /\left(4 Q+2 \sqrt{16+\tilde{Q}^{2} R^{2}}\right)\left(16+\tilde{Q}^{2} R^{2}\right)\left(\rho_{f} / \rho_{s}\right)}{16 C_{1}+8 R^{3} C_{2} \tilde{Q}^{5} /\left(4 \mathrm{Q}+2 \sqrt{16+\tilde{Q}^{2} R^{2}}\right)\left(16+\tilde{Q}^{2} R^{2}\right)\left(\rho_{f} / \rho_{s}\right)}\right]
$$

tion is higher when the anisotropy ratio increases. One possible explanation for this is that the natural rubber foams developed in this work are composed of cells surrounded not only by struts but also by walls and therefore, not only the bending of cell edges but also the stretching of cell faces could play a key role in the deformation mechanisms under compressive stresses of these foams. However, these foams are not the typical closed cell foams because there are a high number of interconnections between cells in the form of holes in the wall (Fig. 5). This was previously described in the cellular structure characterization. Moreover, Huber and Gibson validated this model for polymer foams of low density and with anisotropy ratios between 1 and 1.5. Nevertheless, the anisotropy ratios obtained in this work are even higher than 2 and the foams developed have relative densities between 0.339 and 0.376 . Finally, it is fair to point out that this model considers a very simple geometry of the cell to describe the mechanical behavior of polymer foams.

There is another model in literature in which a more complex cell geometry (tetrakaidecahedron) is considered. This is the case of the model described by Kelvin (Thomson, 1887). Sullivan et al. (2008) (Sullivan and Ghosn, 2009) modified this model by adding a
In this equation: $\tilde{Q}=2+2 Q, C_{1}=3-\pi / 2 \quad$ and $C_{2}=20 \sqrt{3}-$ $11 \pi / 2 \sqrt{3}-\pi$ for a hypocycloid cross section.

In previous works $Q$ was restricted to 1,2 and $\sqrt{2}$ (Sullivan et al., 2008; Sullivan and Ghosn, 2009). In this work the model is represented for these same values and compared with the experimental results and the rectangular cell model. The relative density needed for the model was taken as the average between the density of the different foams produced in this work (NRFI, NRFM and NRFA). The results are shown in Fig. 13.

On the one hand, the experimental values are in good agreement with the theoretical predictions of the Kelvin model in the case of the NRFM and NRFI foams (low anisotropy ratios). This could be due to the more complex geometry employed by this model, which provides a more accurate description of the real cellular morphology of the natural rubber foams developed. Similar results were found in previous works where the stiffness ratio of low density flexible and rigid foams with anisotropy ratios lower than 2 were proved to be in good agreement with the Kelvin model predictions (Hamilton et al., 2013; Sullivan et al., 2008). The value of $Q$ which better described the morphology of the cells is 1.40 in the case of the NRFI foam and 0.93 in the case of the NRFM foam. 
(a)

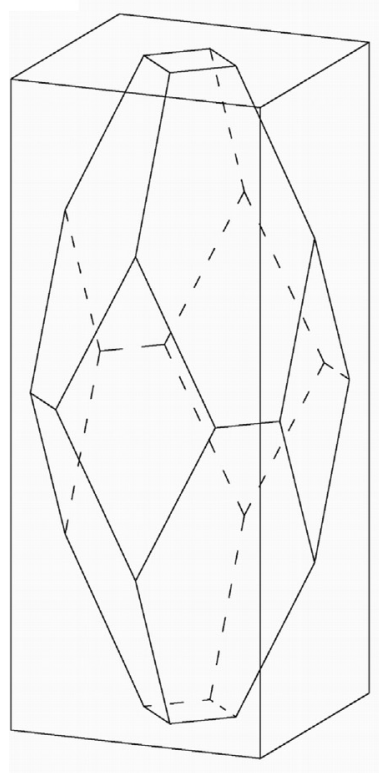

(b)

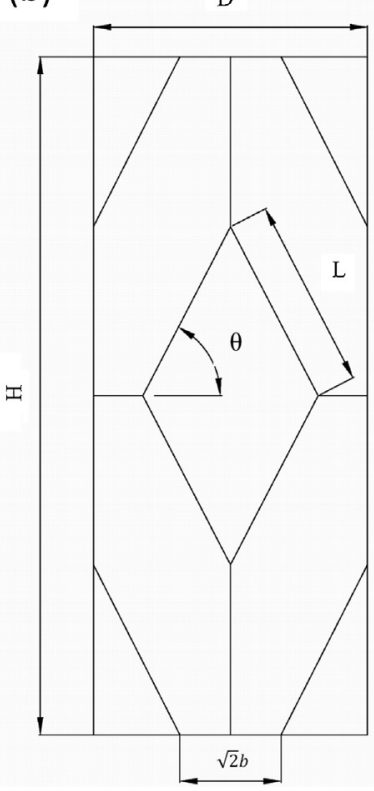

(c)

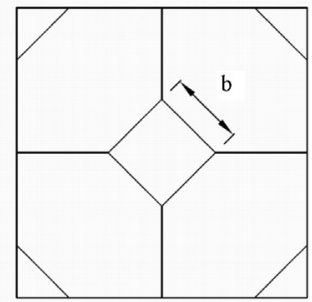

Fig. 12. (a) Rectangular and Kelvin cell model representation in 3D. (b) Front view in 2D. (c) Upper view in $2 D$.

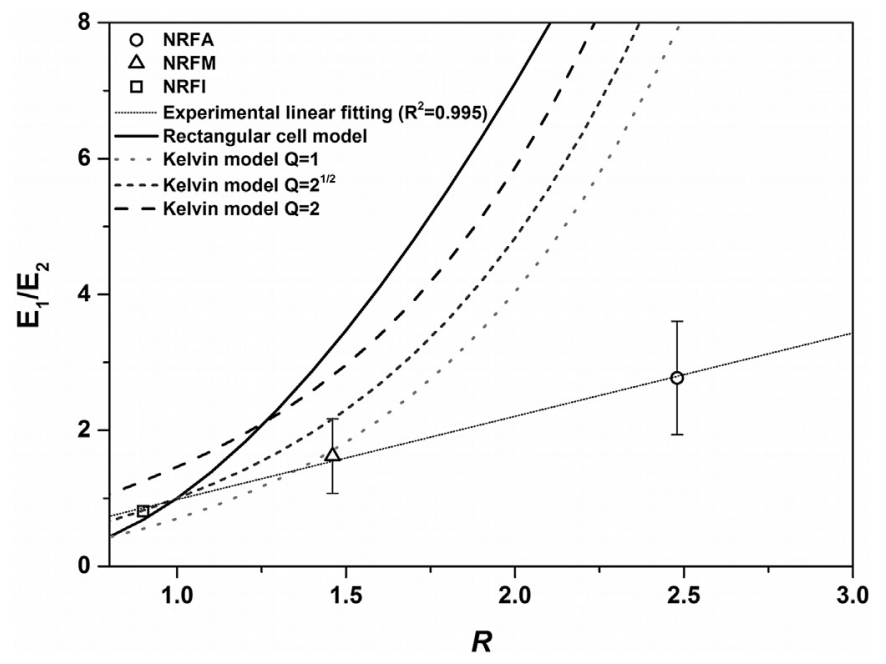

Fig. 13. Theoretical values of the rectangular cell model, Kelvin model and experimental values.

On the other hand, the NRFA foam does not fit well with both the rectangular cell model and the Kelvin model. The experimental stiffness ratio is lower than those obtained for both models. Nevertheless, the difference between the Kelvin model and the experimental value is lower than the difference with respect to the rectangular cell model. Therefore, we can conclude that in the case of flexible foams of medium density and anisotropy ratios higher than 2 such as the natural rubber foams developed in this work the mechanical behavior is not well described by the previous models. In these models the theoretical curves obtained grow exponentially while the experimental results obtained follow a linear tendency as observed in the linear fitting represented in Fig. 13.

\section{Conclusions}

In the first place, natural rubber foams with varied cellular structures regarding anisotropy ratio were obtained by restricting the expansion of the polymer inside a closed mold and by using solid precursors with different dimensions. Moreover, the elastomeric properties of natural rubber and the crosslinking of the polymer matrix during foaming were also of paramount importance to obtain these structures.

Secondly, the cellular structure characterization in the expansion plane by image analysis allowed the quantification of cellular structure parameters such as cell size and cell shape anisotropy ratio. The most significant result is that the shape anisotropy ratio varied from 0.90 to 2.48 . All the foams presented high open cell content.

Geometrical considerations allowed a theoretical anisotropy ratio to be obtained which was compared with the experimental anisotropy ratio. The theoretical prediction fit well for the foams of low anisotropy such as the NRFI and the NRFM foams. On the contrary the theoretical anisotropy ratio of the NRFA foam is higher than the experimental result. This could be due to the fact that cells are more likely to be generated during the anisotropic expansion of this foam because the time in which growth in one direction takes place is higher than in the other two. The generation of new cells during the expansion could avoid the elongation of the cells already created at the beginning of the foaming process reducing the expected anisotropy ratio.

Finally, compression tests were carried out in two directions in all the foams produced and the compressive modulus was calculated from these tests. With regards to the results in the expansion direction the main conclusion is that the higher the anisotropy ratio, the higher the compressive modulus. The experimental results were compared with models found in literature, such as the rectangular cell model and the Kelvin cell model. The experimental results seem to fit well with the Kelvin cell model when low anisotropies are considered. This is the case of the NRFI and NRFM foams. However, when high anisotropies are considered such as in the case of the NRFA foam the experimental result is far removed from both predictions.

\section{Acknowledgements}

This work performed with the financial support from CNPq (Conselho Nacional de Desenvolvimento Científico e Tecnológico-Brasil), MCINN (MAT2009-14001-C02-01 and MAT 
2012-34901), the Junta of Castile and Leon (VA035U13) and FPI grant Ref: BES-2010-038746 (Alberto Lopez-Gil).

\section{References}

Abrahama, E., Thomas, M.S., John, C., Pothen, L.A., Shoseyova, O., Thomas, S., 2013. Green nanocomposites of natural rubber/nanocellulose: membrane transport, rheological and thermal degradation characterizations. Ind. Crop Prod. 51, 415-424, http://dx.doi.org/10.1016/j.indcrop.2013.09.022.

Archer, B.L., 1963. Structure, composition and biochemistry of Hevea latex. In: Bateman, L. (Ed.), The Chemistry and Physics of Rubber-like Substances. Mac Laren \& Sons, London.

Ariff, Z.M., Zakaria, Z., Tay, L.H., Lee, S.Y., 2008. Effect of foaming temperature and rubber grades on properties of natural rubber foams. J. Appl. Polym. Sci. 107, 2531-2538, http://dx.doi.org/10.1002/app.27375.

Bellucci, F.S., Salmazo, L.O., Budemberg, E.R., da Silva, M.R., Rodríguez-Pérez, M.A., Nobre, M.A.L., Job, A.E., 2012. Preparation and structural characterization of vulcanized natural rubber nanocomposites containing nickel-zinc ferrite nanopowders. J. Nanosci. Nanotechnol. 12, 2691-2699, http://dx.doi.org/10. 1166/jnn.2012.5694.

Christensen, R.M., 1986. Mechanics of low density materials. J. Mech. Phys. Solids 34, 563-578, http://dx.doi.org/10.1016/0022-5096(86)90037-2.

Eaves, D., 2004. Handbook of Polymer Foams. Rapra Technology, Shrewsbury.

Ferreira, M., 2002. Evaluation of natural rubber from clones of Hevea brasiliensis. Rubber Chem. Technol. 75, 171-177, http://dx.doi.org/10.5254/1.3547668.

Gibson, L.J., Ashby, M.F., 1997. Cellular Solids: Structure and Properties, second ed. Cambridge University Press, Cambridge.

Gong, L., Kyriakides, S., Jang, W.Y., 2005a. Compressive response of open cell foams. Part I: morphology and elastic properties. Int. J. Solids Struct. 42, 1355-1379, http://dx.doi.org/10.1016/j.ijsolstr.2004.07.023.

Gong, L., Kyriakides, S., Triantafyllidis, N., 2005b. On the stability of kelvin cell foams under compressive loads. J. Mech. Phys. Solids 53, 771-794, http://dx doi.org/10.1016/j.jmps.2004.10.007.

Gupta, S., Watson, B., Beaumont, P.W.R., Ashby, M.F., 1986. Final Year Project. Cambridge University Engineering Department, Cambridge, UK.

Hamilton, A.R., Thomsen, O.T., Madaleno, L.A.O., Jensen, L.R., Rauhe, J.C.M., Pyrz, R., 2013. Evaluation of the anisotropic mechanical properties of reinforced polyurethane foams. Compos. Sci. Technol. 87, 210-217, http://dx.doi.org/10. 1016/j.compscitech.2013.08.013.

Hilyard, N.C., 1982. Mechanics of Cellular Plastic, first ed. Applied Science, London.

Huber, A.T., Gibson, L.J., 1988. Anistropy of polymer foams. J. Mater. Sci. 23, 3031-3040, http://dx.doi.org/10.1007/BF00547486.

Jong, L., 2015. Influence of protein hydrolysis on the mechanical properties of natural rubber composites reinforced with soy protein particles. Ind. Crops Prod. 65, 102-109, http://dx.doi.org/10.1016/j.indcrop.2014.12.004.
Joon-Hyung, K., Kyo-Chang, C., Jin-Min, Y., 2006. The foaming characteristics and physical properties of natural rubber foams: effects of carbon black content and foaming pressure. J. Ind. Eng. Chem. 12, 795-801.

Joon-Hyung, K., Jae-Song, K., Kyo-Chang, C., Jin-Min, Y., Soo-Yeon, K., 2007. Effects of foaming temperature and carbon black content on the cure characteristics and mechanical properties of natural rubber foams. J. Ind. Eng. Chem. 13, 198-205.

Kanokwiroon, K., Teanpaisan, R., Wititsuwannakul, D., Hooper, A.B. Wititsuwannakul, R., 2008. Antimicrobial activity of a protein purified from the latex of Hevea brasiliensis on oral microorganisms. Mycoses 51, 301-307. http://dx.doi.org/10.1111/j.1439-0507.2008.01490.x.

Kumar, V., 1988. Process Synthesis for Manufacturing Microcellular Thermoplastic Parts. PhD. Thesis. Massachusetts Institute of Technology, Cambridge, MA.

Mehta, B.S., Colombo, E.A., 1976. Mechanical properties of foamed thermoplastics. J. Cell. Plast. 12, 59-66, http://dx.doi.org/10.1177/0021955X7601200107.

Najib, N.N., Ariff, Z.M., Manan, N.A., Bakar, A.A., Sipaut, C.S., 2009. Effect of blowing agent concentration on cell morphology and impact properties of natural rubber foam. J. Phys. Sci. 20, 13-25, 10.1.1.516.4653.

Najib, N.N., Ariffa, Z.M., Bakara, A.A., Sipaut, C.S., 2011. Correlation between the acoustic and dynamic mechanical properties of natural rubber foam: effect of foaming temperature. Mater. Design 32, 505-511, http://dx.doi.org/10.1016/j. matdes.2010.08.030.

Pinto, J., Solórzano, E., Rodriguez-Perez, M.A., de Saja, J.A., 2013. Characterization of the cellular structure based on user-interactive image analysis procedures. J. Cell. Plast. 49, 555-575, http://dx.doi.org/10.1177/0021955X13503847.

Sullivan, R.M., Ghosn, L.J., Lerch, B.A., 2008. A general tetrakaidecahedron model for open-celled foams. Int. J. Solid Struct. 45, 1754-1765, http://dx.doi.org/10. 1016/j.ijsolstr.2007.10.028.

Sullivan, R.M., Ghosn, L.J., 2009. Shear moduli for non-isotropic, open cell foams using a general elongated Kelvin foam model. Int. J. Eng. Sci. 47, 990-1001, http://dx.doi.org/10.1016/j.ijengsci.2009.05.005.

Thomson, W., 1887. On the division of space with minimum partitional area. Philos. Mag. 24, 503-514 (Lord Kelvin).

Zhu, H.X., Knott, J.F., Mills, N.J., 1997. Analysis of the elastic properties of open-cell foams with tetrakaidecahedral cells. J. Mech. Phys. Solids 45, 319-343, http:// dx.doi.org/10.1016/s0022-5096(96)00090-7.

Warren, W.E., Kraynik, A.M., 1987. Foam mechanics: the linear elastic response of two dimensional spatially periodic cellular materials. Mech. Mater. 6, 27-37, http://dx.doi.org/10.1016/0167-6636(87)90020-2.

Weller, J.E., Kumar, V., 2010. Solid-state microcellular polycarbonate foams. I. The steady-state process space using subcritical carbon dioxide. Polym. Eng. Sci. 50, 2160-2169, http://dx.doi.org/10.1002/pen.21736.

Wu, Y.P., Ji, M.Q., Qi, Q., Wang, Y.Q., Zhang, L.Q., 2004. Preparation structure, and properties of starch/rubber composites prepared by co-coagulating rubber latex and starch paste. Macromol. Rapid Commun. 25, 565-570, http://dx.doi. org/10.1002/marc.200300125. 